\title{
NOTICING, AUTOMONITORAMENTO E (DES)FOSSILIZAÇÃO: INTERFACES E (DES)CONSTRUÇÕES EM UM ESTUDO DE CASO
}

\author{
NOTICING, SELF-MONITORING AND (DE)FOSSILIZATION: \\ INTERFACES AND (DE)CONSTRUCTIONS IN A CASE STUDY
}

\author{
Fernanda Vieira da Rocha SILVEIRA ${ }^{1}$
}

Resumo: Este artigo tem como objetivo analisar o processo de (des)fossilização de itens gramaticais e lexicais na produção escrita de uma aprendiz adulta através da prática de noticing, aliada à estratégia metacognitiva de automonitoramento. $\mathrm{O}$ aporte teórico da pesquisa foi embasado na teoria da Fossilização (SELINKER, 1972; HAN, 2003) sob o enfoque de Larsen-Freeman (2005), que defende a complexidade da ideia de fim no processo de aprendizagem de línguas adicionais, nas Hipóteses de Noticing (SCHMIDT, 1990) e Output (SWAIN, 1995) e na estratégia metacognitiva do automonitoramento (O'MALLEY; CHAMOT, 1990; OXFORD, 1990). Desse modo, a presente pesquisa é compreendida como um estudo de caso em andamento de natureza quali-quantitativa dividido em quatro fases, cujo corpus apresenta textos produzidos pela participante entre 2013 e março de 2020, além de três textos produzidos na quarta fase do estudo, de cunho qualitativo. Os resultados obtidos sugerem que a prática de noticing, complementada pelo automonitoramento promoveu maior consciência metalinguística na aprendiz e desestabilização de itens gramaticais e lexicais anteriormente em movimento de fossilização.

Palavras-chave: (Des)fossilização. Noticing. Automonitoramento. Língua-alvo.
Abstract:This article aims to analyze the process of (de)fossilization of grammatical and lexical items in an adult learner's written production through the practice of noticing, coupled with the metacognitive strategy of self-monitoring. The theoretical basis relies on the Fossilization Theory (SELINKER, 1972, HAN, 2003) under Larsen-Freeman's (2005) perspective, who defends the complexity of the idea of an end in second language acquisition. It also relies on the Noticing (SCHIMDT, 1990), and the Output (SWAIN, 1995) Hypotheses, and on the metacognitive strategy of self-monitoring (O'MALLEY; CHAMOT, 1990; OXFORD, 1990). Thus, the present research constitutes a qualiquantitative ongoing case study divided into four phases, whose corpus presents texts produced by the participant between 2013 and March 2020, as well as three texts produced in the fourth phase of the study, which is qualitatively oriented. Results suggest that the practice of noticing, complemented by self-monitoring has raised the learner's metalinguistic awareness, and the destabilization of grammatical and lexical items previously moving towards fossilization.

Keywords: (De)fossilization. Noticing. Selfmonitoring. Target language.

1 Universidade do Estado do Rio de Janeiro (UERJ), Rio de Janeiro, Rio de Janeiro, Brasil; ernanda.silveira72@gmail.com; http://orcid.org/0000-0003-4244-0517 
- Noticing, automonitoramento e (des)fossilização: interfaces e (des)construções em um estudo de caso

\section{Introdução}

O termo "Fossilização", introduzido por Selinker (1972), caracteriza-se como um fenômeno que ocorre durante o desenvolvimento do sistema linguístico produzido por aprendizes de uma segunda língua ou língua estrangeira ao longo do processo de aquisição denominado Interlíngua (SELINKER, 1972). Tal sistema contém traços, tanto da língua-alvo, quanto da língua materna do aprendiz, sendo assim um sistema linguístico único, com características de ambos (ELLIS, 1997). A Fossilização, por conseguinte, ocorre quando há persistência de platôs de itens não pertencentes à língua-alvo na interlíngua dos aprendizes, fazendo com que erros nas estruturas gramaticais, lexicais, fonológicas e pragmáticas façam parte da produção oral e escrita (ELLIS, 2008). Corder (1967 apud ELLIS, 2008) estabelece uma diferença entre desvio e erro. Um desvio ocorre quando o aprendiz apresenta uma falha em sua performance, enquanto o erro resulta da falta de conhecimento de determinada regra da língua. Para os fins deste trabalho, o termo "erro" será utilizado de modo a incluir tanto os lapsos durante a performance quanto a falta de conhecimento das regras da língua.

A literatura mais recente acerca da Fossilização (HAN, 2003; LONG, 2003; BIRDSON, 2004; HAN; ODLIN, 2005; TAJEDDIN; TABATABAEIAN, 2017) nos mostra que o fenômeno ainda carece tanto de uma definição unificada quanto de estudos empíricos, dada a sua natureza dicotômica. Han $(2003,2004)$ defende que a fossilização pode ser global, afetando toda a interlíngua, ou local, quando determinados subsistemas (sintaxe, por exemplo) ou um item em especial, como a formação de um tempo verbal podem fossilizar, enquanto outras áreas apresentam progresso. A Fossilização também pode ser entendida como um produto ou um processo. Como produto, configura-se como o estado permanentemente estagnado, nos âmbitos global e local. Da perspectiva de processo, a fossilização não necessariamente requer a ausência de desenvolvimento em uma determinada área, mas reconhece uma tendência à redução do ritmo de desenvolvimento.

Entre outras variáveis, a dificuldade do aprendiz em notar os itens fossilizados presentes na sua produção pode resultar na persistência destes na interlíngua, tornandose automatizados antes que o aprendiz adquira a forma correta (MITCHELL; MYLES, 2004). Desse modo, esses itens encontram-se fora da área de atenção do aprendiz, em cuja interlíngua tem início um processo de estabilização das formas, como postula Schmidt em sua Noticing Hypothesis (1990). Segundo a teoria, o ato de perceber (noticing) exerce um papel fundamental na aprendizagem da língua-alvo, pois se o aprendiz perceber os itens linguísticos, estes se tornariam intake (influxo), ou seja, da parte do input percebida 
pelo aprendiz. Segundo Doughty (2003), o processo de aquisição da língua-alvo é de certa forma regulado pelos itens que entram no campo de atenção do aprendiz e são percebidos e destacados como relevantes no insumo (input) da língua-alvo.

Assim sendo, para que a percepção dos itens destacados no insumo contribua para o desenvolvimento da aprendizagem, defendo que o processo de Noticing seja aliado ao uso da estratégia de aprendizagem de automonitoramento (Self-monitoring). O'Malley e Chamot (1990), assim como Oxford (1990) categorizam o automonitoramento como uma estratégia metacognitiva, um esforço do aprendiz para gerenciar a si mesmo, caracterizada como uma ação na qual o aprendiz percebe o erro e tenta corrigi-lo, e é neste contexto que insiro a presente pesquisa.

Este artigo é fruto de um estudo de caso longitudinal que vem sendo realizado desde 2013, que busca compartilhar o processo de Noticing, Automonitoramento (Selfmonitoring) e (Des)fossilização no caminho trilhado pela participante, uma aprendiz adulta (sênior). Assim, na segunda seção abordo o aporte teórico da pesquisa, em seguida, descrevo o contexto e os procedimentos metodológicos adotados para a realização do trabalho. Na quarta seção, analiso e discuto os dados parciais, visto que a pesquisa se encontra em andamento. Por fim, apresento as considerações finais.

\section{Sobre a fossilização}

A fossilização foi primeiro definida por Selinker (1972) como um fenômeno linguístico no qual estruturas, regras e subsistemas da língua materna do aprendiz tendem a permanecer na sua interlíngua a despeito da idade, instrução recebida e exposição à língua-alvo. Selinker e Lamendella (1978) prosseguiram em seus estudos e perceberam que esse fenômeno emerge na forma de cessação permanente do desenvolvimento da língua-alvo antes do domínio completo das regras e princípios da língua em nível estrutural e discursivo.

Ellis (1985), em contrapartida, apresentou uma outra visão sobre a fossilização, como algo não necessariamente negativo. Segundo o autor, tanto formas incorretas quanto corretas ficam registradas na interlíngua do aprendiz. Estudos mais recentes sobre a fossilização indicam uma maior variedade de definições e descrições do fenômeno (HAN, 2003; LONG, 2003; BIRDSONG, 2004) ou hipótese, como defende Nakuma (1998). Han (2003) estabelece uma distinção entre fossilização global e local. A primeira afeta toda a interlíngua do aprendiz, trazendo a cessação da aprendizagem defendida por Selinker, enquanto a segunda prevê que a fossilização pode ocorrer em determinados aspectos como artigos ou/e construções verbais, enquanto outras áreas permanecem 
- Noticing, automonitoramento e (des)fossilização: interfaces e (des)construções em um estudo de caso

intactas e seguem em evolução. A autora defende que a fossilização pode ser entendida como produto ou processo. Como produto, corresponde ao estado de desenvolvimento totalmente estagnado, já a visão de fossilização como processo não se refere à falta de desenvolvimento em determinada área, e sim à tendência para a diminuição do desenvolvimento do aprendiz.

Han (2004) propõe que o conceito de fossilização seja entendido em nível macroscópico e microscópico. As pesquisas em nível macroscópico buscam explicar as razões pelas quais as crianças, em geral, aprendem línguas mais efetivamente do que os adultos, através de fatores como restrições maturacionais e transferência da língua materna. Já os estudos em nível microscópico investigam fatores individuais e variáveis socioafetivas que fazem com que os aprendizes se diferenciem em proficiência e níveis de fossilização. Tarone (2006) considera os fatores sociais como a raiz do problema da fossilização e defende que a capacidade de noticing, processo essencial para a aprendizagem, é regulada pelo contexto social de interação do aprendiz, consequentemente, estabelecendo uma relação entre o social, o psicológico e o cognitivo (TARONE, 2006 apud LARSENFREEMAN, 2006).

Larsen-Freeman (2005 apud HAN; ODLIN, 2005) aponta para a complexidade da questão da fossilização, dada a natureza fluida das línguas e sugere uma mudança no mapa conceitual ao propor que não existe um estado final, pois não existe um "fim". A autora defende que, na maioria dos termos associados à fossilização como estado permanente, o fracasso ao atingir uma competência quase nativa na L2 e a cessação do desenvolvimento pertencem a uma visão de língua/interlíngua que possui um estado final. Sendo assim, é importante que exista uma agenda de pesquisa na qual trabalhos que envolvam limite e fossilização sejam complementados pelo estudo da ausência de fronteiras da potencialidade, não só para fins teóricos como para sua implicação prática. O presente estudo se insere neste viés, visto que entende a aprendizagem de línguas como um projeto sem fim ou limites, no qual a busca do professor, pesquisador e aprendiz seja manter a aprendizagem viva.

\section{Noticing e automonitoramento}

Na história dos estudos sobre aquisição de segunda língua (SL)/língua estrangeira (LE), ainda permanece controverso se a aprendizagem da SL/LE se dá de modo consciente, com a atenção do aprendizou inconscientemente. Sobre essa questão, Schmidt se envolveu em pesquisas sobre o impacto da consciência no processo de aprendizagem, dentre elas, sua própria experiência ao aprender a língua portuguesa (SCHMIDT; FROTA, 1986). Em 
1990, o pesquisador propôs a Noticing Hypothesis, na qual a consciência sobre a língua e a atenção dinâmica e ativa aos seus componentes colaboram para a internalização da língua e para a construção do intake. Segundo Lewis (2000), o processo de transição entre noticing e intake ocorre através de atividades que propiciem ao aprendiz uma observação mais acurada da SL/LE e garantam que hipóteses sobre a língua-alvo sejam formuladas mais rápida e cuidadosamente. Schmidt (1990) elencou as seguintes características que podem contribuir para a frequência com que o aprendiz perceberá itens no insumo:

- Frequência do encontro com os itens;

- Saliência perceptual dos itens;

- Estratégias de ensino que foquem na atenção do aprendiz;

- Habilidade de processamento individual (relacionada à aptidão para aprender línguas);

- Exigências das tarefas ou a natureza da atividade na qual o aprendiz participa.

Mais tarde, Schmidt (2001) percebeu que o requisito mínimo para o noticing seria atrair a atenção do aprendiz para elementos gramaticais mais relevantes no insumo a um nível bem maior do que um simples limiar de consciência subjetiva. O autor admite a natureza subjetiva da percepção e defende que a atenção e noticing são aliados aos fatores internos do aprendiz (motivação, aptidão, estilos e estratégias de aprendizagem, conhecimento da língua-alvo e habilidade de processamento).

Na literatura de aquisição de segunda língua destacam-se três posições sobre a relação entre atenção e aprendizagem. A primeira, já mencionada, corresponde à Hipótese de Noticing, na qual Schmidt (1990) defende que o processo requer atenção focal e consciência por parte do aprendiz. Na segunda posição, Tomlin e Villa (1994) argumentam que dos três processos interrelacionados de atenção: estado de alerta, orientação e detecção, somente o último é crucial para a aprendizagem. Na terceira posição, Robinson (1995) une as ideias de Schmidt e Tomlin e Villa ao definir noticing como o que é tanto detectado quanto mais tarde é ativado como resultado da distribuição de mecanismos atencionais. Assim, as exigências de tarefas diversas estimulam diferentes tipos de processamento cognitivo adicional.

Swain (1995) alinha-se à hipótese proposta por Schmidt no que tange ao papel da consciência na aprendizagem de línguas. Em sua Output Hypothesis, a autora foca no processo de produção dos aprendizes, e explica que estes podem tanto notar que algo está "faltando" em sua produção, quanto que existe diferença entre sua própria produção 
- Noticing, automonitoramento e (des)fossilização: interfaces e (des)construções em um estudo de caso

e a língua-alvo. Desse modo, enquanto a Noticing Hypothesis pretende explicar a atenção do aprendiz ao insumo, a Output Hypothesis busca entender a atenção do aprendiz à sua produção e o papel que isto exerce no seu desenvolvimento da língua, gerando reflexão por parte do aprendiz, o que resultaria em uma maior consciência metalinguística (BIALYSTOK, 2001; LEVELT, 1989).

O processo reflexivo ativado pelo exercício de noticing tanto no insumo quanto na produção pode desencadear um maior esforço, por parte do aprendiz, para se autogerenciar e assim fazer uso da estratégia metacognitiva de monitoramento (O'MALLEY; CHAMOT, 1990; OXFORD, 1990). Oxford (1990, p. 136, tradução nossa²) define estratégias metacognitivas como "ações que vão além de artifícios puramente cognitivos e que trazem aos aprendizes um meio para coordenarem seus próprios processos de aprendizagem". Para O'Malley e Chamot (1990), as estratégias metacognitivas envolvem planejamento, monitoramento, avaliação e atenção seletiva. Como na definição de Oxford, essas estratégias são consideradas mais complexas do que as outras, pois requerem do aprendiz habilidades de autogerenciamento e comprometimento.

Diversos pesquisadores realizaram trabalhos sobre estratégias de aprendizagem e pouco divergiram em suas taxonomias (WENDEN; RUBIN 1987; O'MALLEY et al., 1985; OXFORD, 1990; STERN, 1992; ELLIS, 1994, entre outros). Para Oxford (1990), as estratégias metacognitivas se subdividem em centrar a aprendizagem, organizar e planejar a aprendizagem e avaliar a aprendizagem. A autora agrupa as estratégias de aprendizagem em duas categorias: estratégias diretas e indiretas. As estratégias diretas envolvem a língua-alvo e incluem estratégias de memória, estratégias cognitivas e estratégias de compensação. As indiretas não envolvem a língua-alvo diretamente na sua implementação, mas dão suporte e gerenciam a aprendizagem da língua. Neste grupo encontram-se as estratégias metacognitivas, estratégias afetivas e as estratégias sociais. Para os propósitos deste trabalho, o foco será na estratégia indireta metacognitiva de monitoramento.

DeacordocomAnderson(1983apudO'MALLEY;CHAMOT,1990), omonitoramento pode ser explicado como uma resposta para a ambiguidade na compreensão da língua quando alguém seleciona uma melhor forma para o significado de uma mensagem baseado nas informações disponíveis. Oxford (1990) defende que o termo "monitorar" significa notar e corrigir. O automonitoramento também ocorre quando o aprendiz busca produzir seu próprio entendimento sobre o conhecimento do qual não tem certeza.

\footnotetext{
2 No original: "actions which go beyond purely cognitive devices, and which provide a way for learners to coordinate their own learning process".
} 
O monitoramento pode ser abordado tanto de maneira retrospectiva quanto prospectiva. A primeira, pertinente à presente pesquisa, pode ser comparada ao processo de autoavaliação, quando o aprendiz revê sua performance em uma tarefa escrita ou oral (KOSTONS; VAN GOG; PAAS, 2012), enquanto na segunda é solicitado ao aprendiz que faça uma previsão da sua performance em uma determinada tarefa ou teste (KORIAT; ACKERMAN; LOCKL; SCHNEIDER, 2009a, 2009b; METCALFE; FINN, 2008; NELSON; DUNLOSKY, 1991).

O referencial teórico que embasa este trabalho forma um ciclo reflexivo no processo de aprendizagem da língua-alvo, na medida em que envolve o aprendiz no movimento de análise de sua produção, na qual pode vir a perceber lacunas e/ou itens a serem reconstruídos de modo mais acurado, tornando a desfossilização possível.

\section{Procedimentos metodológicos}

A presente pesquisa consiste em um estudo de caso de cunho misto que envolve dados quali-quantitativos, pois defende a inclusão do pluralismo ontológico da pós-modernidade e do ecleticismo metodológico (CRESWELL; PLANO CLARK, 2011). Desse modo, adoto a estratégia integrada simultânea (Concurrent Embedded Strategy, CRESWELL, 2009), na qual a coleta de dados tanto quantitativos quanto qualitativos ocorre simultaneamente de forma complementar, a fim de compor a trama que forma o processo de Noticing, automonitoramento e desfossilização na aprendizagem da língua inglesa.

\section{Contexto da pesquisa}

Em 2011, iniciei a coleta dos dados da minha pesquisa de doutorado (SILVEIRA, 2012), na qual investiguei a ressignificação da ansiedade na aprendizagem da língua inglesa sob a luz da Prática Exploratória (ALLWRIGHT; BAILEY, 1991). Na ocasião, conheci a aprendiz de francês Mila (nome fictício), que me fora apresentada pela sua professora de francês após haver compartilhado seu bloqueio em relação à língua inglesa. No mesmo ano, Mila concordou em participar de encontros exploratórios nos quais desenvolvemos entendimentos sobre seus sentimentos, crenças e atitude em relação a aprender inglês.

Em 2013, Mila decidiu reiniciar seus estudos de língua inglesa comigo em aulas particulares semanais. A aprendiz, em sua fase sênior, sempre relatou sentir muita dificuldade para internalizar as regras e o vocabulário da língua inglesa e durante as aulas compartilhou comigo sua angústia com o fato de não se sentir capaz de se comunicar em 
- Noticing, automonitoramento e (des)fossilização: interfaces e (des)construções em um estudo de caso

inglês (como o faz em francês) durante as suas viagens ao exterior. Mila percebe que sua produção oral e escrita, além da sua compreensão auditiva, apresenta diversas lacunas, pois, apesar da instrução gramatical durante as aulas e da realização de diversos exercícios escritos, determinados itens gramaticais e lexicais permanecem incorretos.

A participante apresenta oscilação quanto ao uso da estratégia de monitoramento durante a interação verbal e escrita, já que em alguns momentos as regras gramaticais são empregadas corretamente e em outros não. Consequentemente, existe a possibilidade de esses itens utilizados incorretamente virem a sofrer fossilização. Saliento que a abordagem de ensino utilizada com Mila se insere na perspectiva defendida por Kumaravadivelu (1994) da era pós-método, na qual elementos das diversas correntes metodológicas podem estar presentes na sala de aula conforme as necessidades apresentadas pelos praticantes (professores e aprendizes). Nesse sentido, o feedback corretivo, implícito ou explícito (ELLIS, 2008), é empregado durantes as aulas com Mila de modo a promover maior conscientização em relação à língua-alvo, sem causar ansiedade.

\section{Instrumentos de coleta e análise dos dados}

A pesquisa foi realizada em quatro fases. Parti do questionamento da Mila sobre seu sentimento de estagnação em relação à aprendizagem do inglês e pedi que ela completasse o questionário SILL (Strategy Inventory for Language Learning), desenvolvido por Oxford (1989), com cinquenta itens distribuídos em seis partes que abordam as estratégias de aprendizagem. Ao conversarmos sobre o questionário, a participante teve a oportunidade de perceber que diversas ações que poderiam facilitar seu processo de aprendizagem não fazem parte das estratégias que utiliza, dentre elas, a estratégia metacognitiva de monitoramento, sobre a qual decidimos nos debruçar.

Na segunda fase, reuni textos escritos em inglês pela participante desde 2013, incluindo mensagens de texto trocadas comigo através do aplicativo WhatsApp, entre 2019 e 2020. Realizei a leitura, a identificação e a quantificação percentual dos itens gramaticais e lexicais que apresentavam erros. De acordo com as ocorrências, os itens foram inseridos nas seguintes categorias: "ortografia", "vocabulário", "tempos verbais", "preposições", "estrutura de perguntas", "forma verbal", "artigos", "pronomes", "ordem de palavras", "quantificadores", "conjunções", "voz passiva", "orações condicionais" e "formação do plural". A participante recebeu o material escrito para iniciar o exercício de Noticing, sem as minhas correções, e foi solicitada a identificar as partes com erros e praticar a estratégia de monitoramento através da reformulação dos itens que entraram no seu campo de atenção. 
A terceira fase consistiu na quantificação percentual e catalogação dos itens percebidos e reformulados pela participante em comparação aos meus. Nesta fase iniciei a análise quantitativa dos dados com o objetivo de conhecer os itens que mais entraram no campo de atenção de Mila, aliada à qualidade da reformulação realizada.

Na quarta fase, de cunho qualitativo, foi utilizado o protocolo verbal Think aloud (ERICSSON; SIMON, 1993) com o objetivo de conhecer, através da verbalização, os processos de pensamento contínuo da aprendiz (JASPERS et al., 2004). Desse modo, foi solicitado à Mila que gravasse a realização da tarefa de Noticing e monitoramento de seus textos escritos sobre temas selecionados por ela, com intervalo entre eles após a terceira fase da pesquisa. O primeiro foi entregue no dia dezenove de abril, o segundo no dia vinte e cinco de abril e o terceiro, no dia vinte e nove de abril de 2020. Durante as tarefas de produção e noticing, ficou estabelecido com a participante que não haveria consultas a dicionários ou gramáticas. A gravação foi realizada uma semana depois do envio à pesquisadora do terceiro texto. Integrados à análise quantitativa, os dados obtidos nesta fase buscam responder às seguintes perguntas:

1. Quais e em que proporção categorias gramaticais e lexicais foram percebidas e reformuladas de modo acurado pela participante?

2. Após as atividades de Noticing e monitoramento, houve algum movimento de desfossilização? Em caso afirmativo, quais itens entraram no campo de atenção da participante em maior frequência?

3. Na quarta fase da pesquisa, quais itens gramaticais e lexicais ainda não entraram no campo de atenção da participante?

\section{Análise e discussão dos dados}

Nos dados selecionados para o estudo, os erros que apareceram foram identificados e classificados em quatorze categorias: "ortografia", "vocabulário", "formas verbais", "preposições", "artigos", "tempos verbais", "pronomes", "ordem de palavras", "estruturas de perguntas", "quantificadores", "conjunções", "orações condicionais", "plurais" e "voz passiva". A variedade de categorias corrobora a argumentação de que a fossilização pode ocorrer em diferentes áreas da língua (NOZADZE, 2012; WEI, 2008). No total foram categorizados 451 erros, dos quais a participante percebeu e monitorou 268. A tabela abaixo apresenta o total e o percentual de cada categoria. Serão apresentados os itens identificados pela pesquisadora e os itens percebidos e monitorados pela participante. $\mathrm{O}$ percentual apresentado para os itens percebidos e monitorados durante o exercício de Noticing foram calculados em relação ao total de itens referentes à cada categoria. 
- Noticing, automonitoramento e (des)fossilização: interfaces e (des)construções em um estudo de caso

Tabela 1. Itens observados e itens percebidos e monitorados:

\begin{tabular}{|c|c|c|c|c|}
\hline CATEGORIAS & \begin{tabular}{l|} 
ITENS \\
OBSERVADOS \\
(Total- 451$)$
\end{tabular} & $\begin{array}{l}\text { ITENS } \\
\text { OBSERVADOS } \\
\text { (percentual) }\end{array}$ & $\begin{array}{l}\text { ITENS PERCEBIDOS/ } \\
\text { MONITORADOS } \\
\text { (total- 268) }\end{array}$ & $\begin{array}{l}\text { ITENS } \\
\text { PERCEBIDOS/ } \\
\text { MONITORADOS } \\
\text { (percentual) }\end{array}$ \\
\hline $\begin{array}{l}\text { 1- Ortografia } \\
\text { Ex: "Cold you stop at the next } \\
\text { corner?" }\end{array}$ & 95 & $21,05 \%$ & 72 & $72,78 \%$ \\
\hline $\begin{array}{l}\text { 2- Vocabulário } \\
\text { Ex: "It's a possible" }\end{array}$ & 78 & $17,29 \%$ & 44 & $56,41 \%$ \\
\hline $\begin{array}{l}\text { 3- Formas verbais } \\
\text { Ex: "Don't try learn..." }\end{array}$ & 50 & $11,08 \%$ & 22 & $44 \%$ \\
\hline $\begin{array}{l}\text { 4- Preposições } \\
\text { Ex: "I will tell to you..." }\end{array}$ & 48 & $10,64 \%$ & 24 & $50 \%$ \\
\hline $\begin{array}{l}\text { 5- Artigos } \\
\text { Ex: "I made an appointment with } \\
\text { doctor." }\end{array}$ & 45 & $9,97 \%$ & 17 & $37,77 \%$ \\
\hline $\begin{array}{l}\text { 6- Tempos verbais } \\
\text { Ex: "I have already seen your } \\
\text { message when you called me" }\end{array}$ & 38 & $8,42 \%$ & 30 & $78,94 \%$ \\
\hline $\begin{array}{l}\text { 7- Pronomes } \\
\text { Ex: "I heard that yours focus is..." }\end{array}$ & 28 & $6,20 \%$ & 12 & $42,85 \%$ \\
\hline $\begin{array}{l}\text { 8- Ordem de Palavras } \\
\text { Ex: "...and work with them the } \\
\text { contents" }\end{array}$ & 27 & $5,98 \%$ & 10 & $37,03 \%$ \\
\hline $\begin{array}{l}\text { 9- Estrutura de perguntas } \\
\text { Ex: "There will be fog?" }\end{array}$ & 23 & $5,09 \%$ & 22 & $95,65 \%$ \\
\hline $\begin{array}{l}\text { 10- Quantificadores } \\
\text { Ex: "Have you got some defect or } \\
\text { addiction?" }\end{array}$ & 7 & $1,55 \%$ & 4 & $57,14 \%$ \\
\hline $\begin{array}{l}\text { 11- Conjunções } \\
\text { Ex:"...and Wednesday a full day } \\
\text { Rafael's birthday" }\end{array}$ & 5 & $1,10 \%$ & 5 & $100 \%$ \\
\hline $\begin{array}{l}\text { 12- Orações Condicionais } \\
\text { Ex: "We can return in February if } \\
\text { this will be good for you." }\end{array}$ & 3 & $0,66 \%$ & 2 & $66,66 \%$ \\
\hline $\begin{array}{l}\text { 13- Voz passiva } \\
\text { Ex: "...and as such she must } \\
\text { treated" }\end{array}$ & 3 & $0,66 \%$ & 3 & $100 \%$ \\
\hline $\begin{array}{l}\text { 14-Plural } \\
\text { Ex: “...through unforgettable } \\
\text { persons..." }\end{array}$ & 1 & $0,22 \%$ & 1 & $100 \%$ \\
\hline
\end{tabular}

Fonte: Elaboração própria

O quadro apresentado descreve o panorama inicial do processo de Noticing e monitoramento/correção da participante realizado em março de 2020. Dos 451 itens 
observados pela pesquisadora, Mila foi capaz de perceber e monitorar 268, o que representa 59,42\% do total. Os resultados apontam que a categoria "ortografia" apresentou o maior número de itens incorretos, seguida de "vocabulário" e "formas verbais". Ressalto que a categoria "vocabulário" englobou todas as escolhas lexicais de Mila, quanto a substantivos, adjetivos, advérbios, verbos e expressões idiomáticas. O alto percentual de noticing e correção das categorias plurais, voz passiva e conjunções pode ser atribuído ao baixo número de ocorrências na produção da participante, contribuindo para a entrada destes em seu campo de atenção.

Em relação à ortografia, Mila obteve um alto percentual denoticing e monitoramento e corrigiu $75,78 \%$ dos itens e atribuiu os erros à atenção no momento da produção. Das 78 ocorrências envolvendo "vocabulário", a participante percebeu e corrigiu 44, o que representa 56,41\% do total. Segundo Marefat e Nushi (2012), escolhas semânticas não são diretamente governadas por regras, consequentemente apresentam maior complexidade de correção para o aprendiz, já que este não pode recorrer facilmente às regras adquiridas da língua para evitar este tipo de erro. Ao longo da análise, muitos itens da categoria vocabulário não percebidos pela participante sofreram transferência (SELINKER, 1972) da língua materna (português, doravante L1), como no exemplo: "She is able to make up", no qual Mila recorre ao português e transforma o substantivo make-up em verbo. Em outra ocorrência, a participante descreve um episódio em que compartilhou seu arquivo de vocabulário em inglês com um amigo e escreveu: "I sent him my vocabulary. He was charmed.". Neste excerto, percebe-se que, no momento da produção, Mila buscou no dicionário o adjetivo "encantado" e obteve "charmed", entretanto, não houve uma checagem do uso do adjetivo em relação ao contexto desejado.

Na terceira categoria com maior número de ocorrências, relativamente às formas verbais, foram observados 50 itens, dos quais 22 foram percebidos e corrigidos pela participante, com um percentual de $44 \%$. Dos itens observados, o padrão de erros que mais se repetiu foi o uso de verbos que deveriam ser seguidos de infinitivo, com dezessete ocorrências, como nos exemplos: "I try rewrite the same dialogues.", "Don't try learn English studyng grammar", "I'll try review my summry...", "They want meet us", "I need send you something". Quando este tipo de ocorrência existe durante as aulas, Mila recebe feedback corretivo, no qual a regra de uso dos verbos é lembrada, entretanto, mesmo após realizar exercícios na gramática sobre o tema, os erros persistiam. Com a proposta do exercício de noticing, iniciado em abril de 2020, esses itens começaram a entrar no campo de atenção da aprendiz e ela percebeu e corrigiu dez deles. Após o exercício, percebo em sua fala durante as duas aulas semanais que ela vem monitorando este padrão, apresentando certa desfossilização. Outros itens percebidos e corrigidos pertencem a padrões variados, 
- Noticing, automonitoramento e (des)fossilização: interfaces e (des)construções em um estudo de caso

como verbos frasais seguidos de gerúndio, preposições seguidas de gerúndio e uso da forma base após os modais

Dentre as categorias mais percebidas e monitoradas por Mila, destaco "estruturas de perguntas". Do total de 23 ocorrências, 22 foram percebidas e corrigidas pela participante, o que representou um percentual de 95,65\%. Exemplos como "It's going to be foggy?", "This is your first time here?", ocorridos em 2013; "We can talk about it?" (2019), "It's right?" (feveveiro/2020) e "I'm writing correctly?" (março/2020) apontam para a falta de monitoramento em relação à posição do verbo auxiliar na forma interrogativa. A menção ao ano em que os itens ocorreram demonstra a persistência dos erros apesar do feedback corretivo. Em alguns momentos, Mila consegue se monitorar e produz perguntas acuradas: "Do you miss me?", "Where is it?", "How can I go there?" (2013); "Is the writer's opinion critical?" (2017), "What do you miss?" (2018), "This message was in my cell phone, do you believe? (2019), "Can you speak more about it?" (janeiro/2020), "What do you prefer?" (março/2020). Durante o exercício de noticing, Mila reorganizou quase todas as suas perguntas e posicionou corretamente os verbos auxiliares. Com isso, houve uma desestabilização de uma possível tendência à fossilização no que tange à formação de perguntas na língua-alvo.

Outra categoria altamente percebida e monitorada por Mila foi "tempos verbais", ao corrigir 30 dos 38 itens observados, com o total de 78,94\%. Nas ocorrências observadas, não houve predominância de tempo verbal; foram detectados erros variados, como mostram os excertos: "Do you made your reservation..." (2013), "... this story is told from ${ }^{3}$ a man who has his face deformed and decided..." (2015), "..., today fashion became a fad" (2017), "I have never been using some precious resources;" (março/2020). O primeiro exemplo mencionado apresenta interferência da L1 (SELINKER, 1972), no qual Mila faz uso do verbo principal na forma flexionada e não na forma base acompanhada do auxiliar "did". Os três excertos citados foram percebidos e corrigidos durante o exercício de noticing. No caso da ocorrência "I chose don't have children" (2013), a participante percebeu que havia um erro, mas não utilizou o imperativo e em vez de realizar a reformulação para "I chose not to have...", preferiu a construção "I chose have no children". Neste caso, houve transferência da L1 em 2013 quando Mila associou "don't" ao advérbio de negação "não" logo, no seu raciocínio, "don't have" comporia a construção desejada "não ter". O alto percentual de monitoramento confronta a natureza permanente da fossilização ao apresentar a desestabilização dos itens observados.

3 A parte em itálico corresponde à categoria das preposições, não analisada neste parágrafo. 
As categorias menos percebidas durante o exercício de noticing foram "ordem de palavras", com 37,03\% de monitoramento, seguida de "artigos" (37,77\%) e "pronomes" (42,85\%). Considerando a categoria "ordem de palavras", Mila percebeu e corrigiu dez das vinte e sete ocorrências. A maioria dos itens sofreu interferência da L1, como nos excertos: "I am just browsing through the window shops." (2013), "I like films war very much." (2015), "She asked him if in Brazil there weren't other TV programs" (2016), "You miss me so much." (2018) "...pick up them.., (2019) e "She recommended me it." (2020). No primeiro exemplo, Mila não percebeu o erro em "window shops", no qual percebemos o raciocínio seguindo a ordem da L1 "janela da loja" em vez de "loja janela", como seria em "shop window". No caso do item "films war", a participante percebeu o erro e o monitorou para "war movies" durante o processo de noticing. No terceiro exemplo, houve a percepção do erro e monitoramento para "...asked if there was no other type of program in Brazil.". Durante as aulas, Mila recebeu feedback corretivo acerca do uso do verbo "miss", pois acreditava que significava "fazer falta", assim "You miss me" significaria 'você me faz falta'. Ao longo do exercício de noticing, Mila percebeu e corrigiu todas as ocorrências envolvendo o verbo "miss". As ocorrências "...pick up them" foi monitorada para "pick up the books" e "She recommended me it" para "She recommended it to me".

A análise da categoria "artigos" não mostrou um padrão uniforme de erros. Houve itens que sofreram interferência da L1, como ilustram os excertos: "The reforestation would be..." (2013), "Would you like a dessert?" (2013), "Since I was teenager..." (2014), "Through the photoshop..." (2017), "...and it has made the time pass." (2020) e "Do you have idea...?" (2020). Destes itens, não foram percebidos "a dessert" e "the time". Outras ocorrências demonstraram falta de monitoramento do uso dos artigos na língua-alvo: "They are behaving in an universal way." (2013), "...the shark inside an lake" (2017), "This a interesting thesis" (2020). Mila não percebeu "an universal", pois empregou o conhecimento de que " $a$ " seria utilizado antes de sons de vogais, entretanto, a vogal " $u$ " no adjetivo "universal" apresenta som de consoante (Y), devendo ser precedido por "a" em vez de "an".' Nos demais excertos mencionados, houve monitoramento.

Na categoria "pronomes", Mila percebeu e monitorou doze dos vinte e oito itens observados pela pesquisadora. Houve interferência da L1 no uso dos pronomes na línguaalvo, principalmente no caso de "it": "You will see a sign indicating" (2013), "I make a list, you review" (2018), "...but is my birthday!" (2018), "I think you will love" (2019), "Sometimes is difficult" (2019) e "If is right...". Nos excertos destacados, Mila fez uso da tradução literal para a língua inglesa e durante o exercício de noticing, somente percebeu e inseriu o pronome objeto "it" após "review" e "love". As outras construções permaneceram fora do campo de atenção da aprendiz. Um outro item que se repetiu na produção inicial foi 
- Noticing, automonitoramento e (des)fossilização: interfaces e (des)construções em um estudo de caso

o pronome possessivo "yours" em vez de "your": "I heard that yours focus is..." (2013) e "I'll put yours suggestions..." (2020). O fato de esses pronomes ocorrerem nos extremos do período de produção observado, 2013 e 2020, pode representar uma tendência à fossilização desse item, caso não haja associação entre feedback corretivo e noticing. A referenciação através dos pronomes "he" e "she" compôs a maioria dos itens percebidos e monitorados.

Na quarta fase da pesquisa, Mila escreveu três textos de livre escolha e decidiu produzir sobre suas lembranças. No primeiro texto (19/04/20), escreveu sobre o dia do seu casamento, no segundo (25/04/20) relembrou o momento em que sua avó introduziu peixes na fazenda que possuía e no terceiro texto $(29 / 04 / 20)$ escreveu sobre como conheceu e se apaixonou pelo seu marido. A análise das produções mostrou 41 erros em nove categorias: vocabulário (14), tempos verbais (8), preposições (3), pronomes (4), ortografia (4), artigos (3), quantificadores (2), formas verbais (2) e ordem de palavras (1). Durante o exercício de noticing, Mila percebeu e corrigiu 21 itens.

Sobre o excerto seguinte, do primeiro texto, Mila comentou durante a gravação do "think aloud": "... aqui eu acho que ficou legal... não mexeria": "It was a beautiful day. I wake up early and the sun was starting to shine. My dad brought me my last single breakfast in bed, and while I had my breakfast we talked a lot. Together we relive my life, my childhood, my adolescence, my loves and, finally, he talked about my new life, an adult life, and my responsibilities as woman."

Mila inicia a narrativa com um verbo no passado "was" e utiliza outras construções também no passado como "brought", "had" e "talked". Contudo, durante o exercício de noticing, não percebeu dois verbos que não estavam conjugados como os outros: "wake up" e "relive". Em todo o texto, estes foram os únicos verbos conjugados incorretamente. Outro item não percebido por Mila foi o uso do adjetivo "single" em "single breakfast". Sua intenção era dizer "café da manhã de solteira", o que sugere interferência da L1. No terceiro parágrafo, ao prosseguir com a leitura do texto, Mila percebeu certa discrepância com a língua-alvo no termo "review of luggage": "Eu acho que isso aqui tá meio artificial, eu acho que luggage check fica melhor... não sei... vou trocar". Na gravação, Mila percebeu e corrigiu alguns itens sem expressar comentários, logo não farão parte do corpo da análise. A categoria "ortografia" apresentou significativa melhora em comparação com a primeira fase dos dados (entre 2013 e março de 2020), com apenas quatro ocorrências nos três textos, sendo que todos os itens foram percebidos e corrigidos durante o processo de noticing. No último parágrafo, Mila percebeu o erro na palavra "faire" em "faire tale" e comentou: "[...] pera aí, eu acho que fairy é com ' $y$ ', aqui eu errei a escrita". 
No segundo texto (25/04/20), houve treze ocorrências, das quais dez foram percebidas e corrigidas. O primeiro excerto destacado da gravação ilustra o monitoramento das construções "would be owns them" e "destiny": "We would be owns them (leitura lenta seguida de pausa) meu pai! Nós seríamos os donos deles, eles não seriam [...] their owners, donos deles, dos peixes, their owners, and we would also be responsible for their food and destiny, their food and fate, destiny é direção, destino de vida é fate". Mila ponderou e percebeu que algo não "soa" bem na construção "owns them" e reformulou o trecho corretamente. Já em relação à troca do substantivo "destiny" por "fate", ela o confundiu semanticamente com "destination", cuja denotação remete à localização, direção, como mencionou. A escolha lexical "fate", por conseguinte, foi mais adequada à intenção comunicativa do excerto.

No seguinte excerto, "But there was a day that they become many and we were forced to take them to the dam", Mila comentou: "[...] mas houve um dia, aqui seria no qual ou quando, which ou when, when, when they become tá errado, é passado, became muito (pausa), eles se tornaram muitos, então so many, and we were forced to take them to the dam (pausa), that, tira o and.".

Primeiramente, Mila percebeu algo errado em "that" e se decidiu por "when", em seguida monitorou o verbo "become", conjugando-o no passado. O quantificador "many" também sofreu reformulação com a inclusão do advérbio "so" e do determinante "that". Um item que permaneceu fora do campo de atenção da participante foi o uso do infinitivo negativo. No trecho "We decided don't have fishes anymore [...]", Mila não percebeu o erro devido à transferência de uso do auxiliar negativo "don't" para outros contextos com conotação negativa.

O terceiro texto produzido apresentou onze ocorrências com quatro correções. O trecho destacado mostra o caminho percorrido por Mila para a construção "There is all started." para expressar a ideia "Lá tudo começou", quando descrevia o início do relacionamento com seu marido: "There is all started (pausa), isso aqui não tá legal, was all started? Tava tudo começado? Não sei, isso aqui não. (pausa) eu acho que encontrei a solução, there, everything had started.".

Mila percebeu a discrepância com a língua-alvo e reformulou o trecho com mais coerência, apesar do posicionamento incorreto do advérbio de lugar "there". Houve monitoramento da preposição no excerto "[...] When we were dancing Rafael stepped my dress [...]" e Mila comentou: "[...] Quem pisa, pisa em alguma coisa, tá faltando uma preposição aqui, pisar sobre, on.". Nesta ocorrência, nota-se a interferência da L1 quando Mila transfere a regra de regência do verbo pisar para a língua-alvo, quando disse "quem 
- Noticing, automonitoramento e (des)fossilização: interfaces e (des)construções em um estudo de caso

pisa, pisa em alguma coisa" e obteve sucesso na correção pelo fato de as duas línguas requisitarem o uso da preposição.

Apesar de haver, nas três produções, maior monitoramento em relação ao uso dos tempos verbais, ainda existem lapsos de atenção, como no seguinte excerto: "Well, this date was finally over, and I'm free again.", no qual Mila demonstrou conhecimento sobre a conjugação do verbo "be" no passado ao utilizar "was", entretanto não monitorou a construção "I'm", apesar de narrar um evento que ocorreu no passado.

Os quadros a seguir sintetizam a quarta fase da pesquisa apresentando as categorias contendo erros, suas respectivas ocorrências e monitoramentos:

Quadro 1. Produção $1(19 / 04 / 20)$

\begin{tabular}{|c|c|c|}
\hline CATEGORIAS & OCORRÊNCIAS & MONITORAMENTOS \\
\hline VOCABULÁRIO & $\begin{array}{l}\text { Single breakfast } \\
\text { Each other's hand } \\
\text { Review of luggage } \\
\text { Besides }\end{array}$ & $\begin{array}{l}\text { Não houve noticing } \\
\text { Não houve correção } \\
\text { *luggage check } \\
\text { *apart from }\end{array}$ \\
\hline TEMPOS VERBAIS & $\begin{array}{l}\text { Wake up } \\
\text { Relive } \\
\text { Passed } \\
\text { Leave }\end{array}$ & $\begin{array}{l}\text { Não houve noticing } \\
\text { Não houve noticing } \\
\text { Não houve noticing } \\
\text { *leaving }\end{array}$ \\
\hline PREPOSIÇÕES & (arriving) to & $*$ from \\
\hline PRONOMES & $\begin{array}{l}\text { Was the time } \\
\text { On honeymoon }\end{array}$ & $\begin{array}{l}\text { *it was } \\
\text { Não houve noticing }\end{array}$ \\
\hline ARTIGOS & $\begin{array}{l}\text { As woman } \\
\text { One dearest }\end{array}$ & $\begin{array}{l}\text { Não houve noticing } \\
\text { Não houve noticing }\end{array}$ \\
\hline ORTOGRAFIA & $\begin{array}{l}\text { Felling } \\
\text { Faire }\end{array}$ & $\begin{array}{l}\text { Não houve noticing } \\
\text { *fairy }^{\text {fain }}\end{array}$ \\
\hline FORMA VERBAL & Wanting let & Não houve noticing \\
\hline ORDEM DE PALAVRAS & Lunch family & Não houve noticing \\
\hline
\end{tabular}

Fonte: Elaboração própria

Algumas ocorrências não foram percebidas por Mila devido à interferência da L1, como em "single breakfast" (café da manhã de solteira), "on honeymoon" (em lua-demel), "as woman" (como mulher) e "lunch family" (almoço de família). A ocorrência "single breakfast" esboça um certo conhecimento por parte da aprendiz em relação à posição dos modificadores na língua-alvo, o que não utilizou na construção "lunch family". 
Quadro 2. Produção $2(25 / 04 / 20)$

\begin{tabular}{|l|l|l|}
\hline CATEGORIAS & OCORRÊNCIAS & MONITORAMENTOS \\
\hline VOCABULÁRIO & Presented & *introduced \\
& Saw & *watched \\
& Owns & *wners \\
& Destiny & *ate \\
& Did a party & *epared a party \\
\hline TEMPOS VERBAIS & Become & *became \\
& Grow up & Não houve noticing \\
\hline PRONOMES & Them & *their \\
& your & *our \\
\hline ORTOGRAFIA & Then & *them \\
\hline FORMA VERBAL & (we decided) don't have & Não houve noticing \\
\hline QUANTIFICADORES & All & *all of them \\
& many & *so many \\
\hline
\end{tabular}

Fonte: Elaboração própria

Em comparação ao exercício de noticing sobre o primeiro texto, Mila apresentou maior percepção e monitoramento das ocorrências ao analisar a produção 2. Desse modo, houve aprimoramento da consciência metalinguística da aprendiz, em especial das consciências sintática e pragmática, percebido ao longo das inserções reflexivas da participante durante a gravação do think aloud.

Quadro 3. Produção 3 (29/04/20)

\begin{tabular}{|c|c|c|}
\hline CATEGORIAS & OCORRÊNCIAS & MONITORAMENTOS \\
\hline VOCABULÁRIO & $\begin{array}{l}\text { Godparents } \\
\text { All started } \\
\text { Anywhere } \\
\text { Took (a little piece) } \\
\text { Showing off }\end{array}$ & $\begin{array}{l}\text { Não houve noticing } \\
\text { *everything had started } \\
\text { Não houve noticing } \\
\text { Não houve noticing } \\
\text { *showing who he was }\end{array}$ \\
\hline TEMPOS VERBAIS & $\begin{array}{l}\mathrm{Had} \\
\mathrm{I} \text { 'm }\end{array}$ & $\begin{array}{l}\text { Não houve noticing } \\
\text { Não houve noticing }\end{array}$ \\
\hline ARTIGOS & Partner & *a partner \\
\hline ORTOGRAFIA & $\mathrm{Al}$ & *he also \\
\hline PREPOSIÇÕES & $\begin{array}{l}\text { As } \\
\text { Stepped my dress }\end{array}$ & $\begin{array}{l}\text { Não houve noticing } \\
\text { *stepped on my dress }\end{array}$ \\
\hline
\end{tabular}

Fonte: Elaboração própria 
- Noticing, automonitoramento e (des)fossilização: interfaces e (des)construções em um estudo de caso

O terceiro texto foi o que apresentou menos categorias com erros, sugerindo maior monitoramento no momento da produção. Houve interferência da L1 na escolha lexical "godparents" (padrinhos) através da tradução literal. Mila transferiu seu conhecimento cultural de casamentos no Brasil, nos quais existem os "padrinhos" para o âmbito cultural da língua-alvo, sem levar o contexto em consideração.

A categoria "vocabulário" superou as demais em número de ocorrências, correspondendo a 34,14\% do total, seguida de "tempos verbais" com 19,51\%. Contudo, houve mais percepção e monitoramento do vocabulário $(64,28 \%)$ do que dos tempos verbais (25\%), logo o uso dos tempos verbais ainda oscila no campo de atenção da aprendiz.

\section{Considerações finais}

Este estudo de caso analisou o caminho percorrido pela aprendiz Mila no processo de percepção e automonitoramento de construções gramaticais e lexicais destoantes da língua-alvo, tendo como corpus textos produzidos em língua inglesa pela participante entre 2013 e 2020. A recorrência de erros em diversas categorias gramaticais e lexicais em diferentes momentos da produção sugere uma tendência à fossilização desses itens. Entretanto, o processo de Noticing aliado ao uso da estratégia metacognitiva de automonitoramento provocou um movimento de desestabilização na interlíngua da aprendiz, o que sugere possível desfossilização desses itens gramaticais e lexicais e corrobora a perspectiva de Larsen-Freeman (2005) acerca do caráter ilimitado da aprendizagem de LEs.

Considerando a primeira pergunta da pesquisa, os resultados mostraram diferença entre a análise dos textos produzidos entre 2013 e março de 2020 e a dos três textos produzidos após a primeira fase do exercício de noticing e monitoramento. Quando praticou noticing pela primeira vez, as categorias percebidas e monitoradas com maior frequência foram: plurais/voz passiva e conjunções (100\%), estruturas de perguntas (95,65\%), tempos verbais $(78,94 \%)$, ortografia $(75,78 \%)$, orações condicionais $(66,66 \%)$, quantificadores $(57,14 \%)$ e vocabulário $(56,41 \%)$. Na análise dos textos produzidos em abril de 2020, menos categorias apresentaram erros, das quais as mais percebidas e monitoradas foram: quantificadores $(100 \%)$, ortografia e pronomes $(75 \%)$, preposições (66,66\%) e vocabulário (64,28\%).

Em resposta à segunda pergunta, após as atividades de noticing e monitoramento, houve movimento de "desfossilização", percebido nos três textos produzidos por Mila na quarta fase da pesquisa. As produções entre 2013 e março de 2020 apresentaram 
"ortografia" como a categoria com maior número de ocorrências (21,05\%), seguida de "vocabulário" (17,29\%) e"formas verbais" (11,08\%). Com a prática da estratégia metacognitiva de automonitoramento durante a produção dos textos da quarta fase do estudo, o número de ocorrências em "ortografia" caiu para 9,75\% do total, o que sugere maior atenção a esta categoria. O uso das "formas verbais" também passou a sofrer mais monitoramento, visto que nos três textos produzidos na última fase houve somente duas ocorrências que envolveram o uso do infinitivo. Outras categorias que apresentaram poucas ocorrências na quarta fase foram: "ordem de palavras", "quantificadores", "preposições", "artigos" e "pronomes".

A produção dos textos na quarta fase da pesquisa, após o extenso corpus analisado ao longo da prática de noticing e monitoramento trouxe à tona alguns itens gramaticais e lexicais que ainda permanecem fora do campo de atenção da aprendiz. Os dados sugerem que Mila recorre à L1 em suas escolhas lexicais ao realizar por diversas vezes a tradução literal dos termos desejados, fazendo com que "vocabulário" configure como a categoria com maior ocorrência. Percebe-se que a interferência da L1 leva Mila a não perceber discrepâncias entre a sua interlíngua e a língua-alvo. A categoria "tempos verbais" ainda apresenta oscilação em relação à atenção. Após "vocabulário", apresentou mais ocorrências na quarta fase, das quais somente $25 \%$ foram percebidas e monitoradas, indicando a necessidade de mais intenso feedback corretivo, aliado a atividades que promovam conscientização acerca da língua-alvo (THORNBURY, 1997), especialmente no movimento entre input (insumo) e intake (influxo).

A análise dos textos durante a quarta fase foi realizada com a utilização do método "think-aloud" (pensar alto). A verbalização proporcionada por esse método pode constituir uma estratégia eficaz para a composição dos textos, pois ao externar suas reflexões e escolhas gramaticais e lexicais, pode haver maior processamento metacognitivo e mais efetiva resolução de problemas (DOMINOWSKI, 1998). Por conseguinte, este estudo sugere que mesmo se um item percebido não for resolvido corretamente, tal experiência pode levar à percepção de informações relevantes em futuros insumos.

Devido ao fato de este estudo de caso envolver somente uma participante, considero que os resultados discutidos não constituem respostas definitivas, especialmente por se tratar de uma pesquisa em andamento. Destarte, a maior implicação deste estudo diz respeito ao desenvolvimento da consciência metalinguística dos aprendizes adultos através da prática de noticing aliada à estratégia metacognitiva do automonitoramento. Pretendo, assim, a partir desta pesquisa, contribuir para a aprendizagem de aprendizes adultos ao enfatizar a relevância do uso de estratégias de aprendizagem para que haja apropriação e reflexão sobre o processo de aquisição da língua-alvo através de uma perspectiva não-limitadora, como propõem os estudos sobre a Fossilização. 
- Noticing, automonitoramento e (des)fossilização: interfaces e (des)construções em um estudo de caso

\section{Referências}

ALLWRIGHT, D.; BAILEY, K. M. Focus on the Language Classroom. Cambridge: Cambridge University Press, 1991

BATEN, K.; HOFMAN, F.; LOEYS, T. Cross-linguistic activation in bilingual sentence processing: The role of word class meaning. Bilingualism: Language and Cognition, v. 14, n. 3, p. 351-359, 2011.

BIRDSONG, D. Second language acquisition and ultimate attainment. In: DAVIES, A; ELDER, C. (ed.). The Handbook of Applied Linguistics. Malden, MA: Blackwell. 2004. p. 82-104.

BIALYSTOK, E. Bilingualism in development: Language, literacy, and cognition. New York: Cambridge University Press, 2001.

COOKE, S. D. Examining transcription, autonomy and reflective practice in language development. RELC Journal, v. 44, n. 1, p. 75-85, 2013.

CRESWELL, J.; PLANO CLARK, V. Designing and conducting mixed methods research. 2. ed. Thousand Oaks, CA: Sage. 2011.

DOUGHTY, C. Instructed SLA. In: DOUGHTY, C.; LONG, M. (ed.). The handbook of second language acquisition. Oxford: Blackwell Publishing Ltd., 2003. p. 256-310.

ELLIS, R. The study of second language acquisition. Oxford: Oxford University, Press, 2008.

ELLIS, R. Second language acquisition. Oxford: OUP, 1997.

ELLIS, R. A theory of instructed second language acquisition. In: ELLIS, N. (ed.). Implicit and explicit learning of languages. London: Academic Press, 1994.

ELLIS, R. Understanding Second Language Acquisition. Oxford: Oxford University Press, 1985.

ERICSSON, A.; SIMON, H. Protocol analysis: verbal reports as data. MIT Press, 1993. 
HAN, Z. Fossilization: Five central issues. International Journal of Applied Linguistics, v. 14, n. 2, p. 212-242, 2004.

HAN, Z. Fossilization: From Simplicity to Complexity. International Journal of Bilingual Education and Bilingualism, v. 6, n. 2, p. 95-128, 2003.

HAN, Z.; ODLIN, T. Studies of fossilization in second language acquisition. Clevedon: Multilingual Matters, 2005.

JASPERS, M.; STEEN, T.; VAN DEN BOS, C.; GEENEN, M. The think aloud method: a guide to user interface design. International Journal of Medical Informatics, v. 73, p. 781-795, 2004.

KORIAT, A.; ACKERMAN, R.; LOCKL, K.; SCHNEIDER, W. The easily learned, easily remembered heuristic in children. Cognitive Development, v. 24, p. 169-182, 2009a.

KORIAT, A.; ACKERMAN, R.; LOCKL, K.; SCHNEIDER, W. The memorizing effort heuristic in judgments of learning: A developmental perspective. Journal of Experimental Child Psychology, v. 102, p. 265-279, 2009 b.

KOSTONS, D.; VAN GOG, T.; PAAS, F. Training self-assessment and task-selection skills: A cognitive approach to improving self-regulated learning. Learning and Instruction, v. 22, p. 121-132, 2012.

KRAMSCH, C.; A'NESS, F.; LAM, E. Authenticity and authorship in the computermediated acquisition of L2 literacy. Language Learning \& Technology, v. 4, p. 78-104. Retrieved February 4, 2003.

KUMARAVADIVELU, B. The Postmethod Condition: (E)merging strategies for Second/ Foreign Language Teaching. TESOL Quarterly, v. 28, n. 1, p. 27-47, 1994.

LARSEN-FREEMAN, D. Second language acquisition and the issue of fossilization: There is no end, and there is no state. In: HAN, Z. H.; ODLIN, T. (ed.). Studies of fossilization in second language acquisition. Clevedon: Multilingual Matters, 2005. p. 189-200.

LEVELT, W. Speaking: From intention to articulation. Cambridge, MA: MIT Press, 1989. 
- Noticing, automonitoramento e (des)fossilização: interfaces e (des)construções em um estudo de caso

LEWIS, M. Language in the lexical approach. In: LEWIS, M. (ed.). Teaching collocation: Further developments in the Lexical Approach. Hove: Language Teaching Publications, 2000. p. 126-154.

LONG, M. H. Stabilization and fossilization in interlanguage development. In:

DOUGHTY, C.; LONG, M. (ed.). Handbook of second language acquisition. Oxford: Blackwell, 2003. p. 487-536.

MAREFAT, F.; NUSHI, M. Combating Fossilization through Feedback: Does it Work? Iranian Journal of Applied Linguistics (IJAL), v. 15, n. 1, p. 43-71, 2012.

METCALFE, J.; FINN, B. Familiarity and retrieval processes in delayed judgments of learning. Journal of Experimental Psychology: Learning, Memory, and Cognition, v. 34, p. 1087-1097, 2008.

MITCHELL, R.; MYLES, F. Second language learning theories. London: Arnold, 2004.

NAKUMA, C. A new theoretical account of 'fossilization': Implications for L2 attrition research. IRAL. International Review of Applied Linguistics in Language Teaching, v. 36, n. 3, p. 247-256, 1998.

NELSON, T. O.; DUNLOSKY, J. When people's judgments of learning (JOLs) are extremely accurate at predicting subsequent recall: The "delayed-JOL effect". Psychological Science, v. 2, p. 267-270, 1991.

O'MALLEY, J. et al. Learning Strategy Applications with Students of English as a Second Language. TESOL Quarterly, v. 19, p. 557-584, 1985.

O'MALLEY, J. M.; CHAMOT, A. U. Learning strategies in second language acquisition. Cambridge, UK: Cambridge University Press, 1990.

OXFORD, R. Language Learning Strategies: What Every Teacher Should Know. New York: Newbury House Publishers, 1990.

RICHARDS, J.; PLATT, J. Longman Dictionary of Language Teaching and Applied Linguistics. Essex: Longman, 1992. 
ROBINSON, P. Attention, memory, and the "noticing" hypothesis. Language Learning, v. 45, p. 283-331, 1995.

SCHMIDT, R. W. Attention. In: ROBINSON, P. (ed.). Cognition and second language instruction. Cambridge: Cambridge University Press, 2001. p. 3-32.

SCHMIDT, R. W. The role of consciousness in second language learning. Applied Linguistics, v. 11, n. 2, p. 129-158, 1990.

SCHMIDT, R.; FROTA, S. Developing basic conversational ability in a second language: a case study of an adult learner of Portuguese. In: DAY, R. (ed.). Talking to Learn: Conversation in Second Language Acquisition. Rowley, MA: Newbury House, 1986.

SELINKER, L. Interlanguage. IRAL, v. 10, n. 3, p. 209-231, 1972.

SELINKER, L.; LAMENDELLA, J. T. Two perspectives on fossilization in interlanguage learning. Interlanguage Studies Bulletin, v. 3, n. 2, p. 143-191, 1978.

SILVEIRA, F. V. R. Ressignificando a ansiedade na aprendizagem de línguas estrangeiras através das crenças: um estudo exploratório. 2012. Tese (Doutorado em Letras) Pontifícia Universidade Católica do Rio de Janeiro, Rio de Janeiro, 2012.

STERN, H. H. Issues and Options in Language Teaching. Oxford: OUP, 1992.

SWAIN, M. Three functions of output in second language learning. In: COOK, G.; SEIDLHOFER, B. (ed.). Principle and Practice in Applied Linguistics studies in Honour of H.G. Widdowson. Oxford University Press, 1995. p. 125-144.

TAJEDDIN, Z.; TABATABAEIAN, M. S. Interface between linguistic noticing and fossilization of grammatical, lexical and cohesive features among advanced EFL learners. Applied Research on English Language, v. 6, n. 1, p. 23-42, 2017.

TARONE, E. Fossilization, social context and language play. In: HAN, Z. H.; ODLIN, T. (ed.). Studies of fossilization in second language acquisition. Clevedon: Multilingual Matters, 2006. p. 157-172. 
- Noticing, automonitoramento e (des)fossilização: interfaces e (des)construções em um estudo de caso

TOMLIN, R.; VILLA, V. Attention in cognitive science and second language acquisition. Studies in Second Language Acquisition, v. 16, p. 183-203, 1994.

WENDEN, A.; RUBIN, J. Learner Strategies in Language Learning. New Jersey: Prentice Hall, 1987.

WILLIAMS, M.; BURDEN, R. Psychology for Language Teachers: A Social Constructivist Approach. Cambridge: CUP, 1997.

COMO CITAR ESTE ARTIGO: SILVEIRA, Fernanda Vieira da Rocha. Noticing, automonitoramento e (des)fossilização: interfaces e (des)construções em um estudo de caso. Revista do GEL, v. 17, n. 3, p. 280-303, 2020. Disponível em: https://revistadogel.gel.org.br/

DOI: http://dx.doi.org/10.21165/gel.v17i3.2978

Submetido em: 09/10/2020 | Aceito em: 30/10/2020. 